\title{
La tolleranza glicidica nell'insufficienza renale cronica
}

\author{
A.M. Ciciani ${ }^{1}$, F. Bergesio ${ }^{3}$, G. Monzani ${ }^{1}$, R. Ciuti², F. Martinelli ${ }^{3}$, R. Piperno ${ }^{3}$, \\ G. Baldereschi ${ }^{4}$, M. Salvadori \\ ${ }^{1}$ UO di Nefrologia e Dialisi, \\ ${ }^{2}$ UO di Chimica Clinica, Ospedale di Torregalli, Azienda USL 10 di Firenze \\ ${ }^{3} \mathrm{UO}$ di Nefrologia e Dialisi, Ospedale di Careggi, Firenze \\ ${ }^{4} \mathrm{UO}$ di Geriatria, Università degli Studi di Firenze
}

n corso di insufficienza renale cronica (IRC) è stata universalmente messa in evidenza una intolleranza glicidica, conseguenza sia della resistenza tissutale periferica all'azione dell'insulina sia di una inappropriata secrezione dell'ormone (1-3).

Importanti chiarimenti sul metabolismo glicidico nell'uremia sono scaturiti dall'introduzione delle tecniche del 'clamp' normo ed iperglicemico (4). Fisiologicamente la risposta insulinica è bifasica: la prima precoce è indicatrice della sensibilità al glucosio delle beta-cellule pancreatiche seguita da una seconda risposta tardiva che dipende dalla sensibilità tissutale periferica all'insulina stessa. Nell'uremia la risposta tardiva è quasi sempre alterata (1), mentre la risposta precoce può essere variabile: diminuita, ritardata, normale o aumentata (10).

In pratica spesso i livelli di glicemia a digiuno dei pazienti uremici sono normali o lievemente aumentati e al test da carico di glucosio solo alcuni mostrano una chiara intolleranza glicidica. Infatti in alcuni pazienti si riscontra un aumento di secrezione insulinica dalle beta cellule tale da compensare la resistenza tissutale periferica e quindi da mantenere una tolleranza glicidica, in altri si riscontrano livelli di insulina normali o bassi e mostrano un'intolleranza glicidica $(1,6)$. Quindi nella IRC l'intolleranza glicidica si svilupperebbe solo quando la risposta delle beta-cellule pancreatiche è così marcatamente compromessa da non poter garantire livelli insulinemici tali da superare l'alterata sensibilità all'ormone dei tessuti periferici (7).

Studi clinici (8) e sperimentali $(9,11)$ hanno suggerito che l'iperparatiroidismo secondario allo stato uremico sarebbe responsabile della riduzione della secrezione d'insulina delle cellule pancreatiche e quindi potrebbe indurre l'intolleranza glicidica.

Pochi dati si possono ricavare dalla letteratura sulle alterazioni del metabolismo glicidico nei vari stadi dell'IRC (15), molti di più riguardano pazienti con IRC grave $o$ in terapia dialitica (19-23).

Scopo del presente studio è stato quello di esaminare la risposta al test da carico orale di glucosio in pazienti affetti da IRC di vario grado in terapia conservativa e di valutare l'eventuale influenza del diverso regime dietetico (dieta vegetariana supplementata con chetoanaloghi vs dieta ipoproteica convenzionale) e dell'iperparatiroidismo secondario sulla tolleranza glicidica nell'uremia.

\section{Materiali e metodi}

Per lo studio sono stati selezionati 45 pazienti affetti da IRC (creatininemia -Cr-
$>1.5 \mathrm{mg} / \mathrm{dl}), 26$ maschi e 19 femmine, di età compresa tra i 35 e i 75 anni (età media $60 \pm 10$ aa). Dodici volontari sani, 8 maschi e 4 femmine, di età compresa tra i 37 e i 63 anni (età media $47 \pm 8$ aa) con funzione renale normale $(\mathrm{Cr}<1.1$ $\mathrm{mg} / \mathrm{dl}$ ) hanno costituito il gruppo di controllo. Sono stati esclusi i soggetti con familiarità diabetica o diabete mellito, con Indice di Massa Corporea (IMC) $>30$, con proteinuria $>1.5 \mathrm{~g} / 24$ ore e quelli in terapia con glicocorticoidi o altri farmaci noti per avere una influenza sul metabolismo glicidico.

I pazienti sono stati divisi in 3 gruppi a seconda del grado di IRC: I) $\mathrm{Cr}>1.5$ - $\leq$ $3 \mathrm{mg} / \mathrm{dl}$; II) $\mathrm{Cr}>3$ - $\leq 6 \mathrm{mg} / \mathrm{dl}$; III) $\mathrm{Cr}>$ $6 \mathrm{mg} / \mathrm{dl}$. Fino a valori di $\mathrm{Cr}<2.5 \mathrm{mg} / \mathrm{dl}$, i pazienti non seguivano alcuna dieta, mentre se la $\mathrm{Cr}$ superava tale valore veniva prescritta la dieta ipoproteica convenzionale (DC) cioè $0.60 \mathrm{~g} / \mathrm{kg} / \mathrm{die}$. Un altro gruppo (IV-DVS) di pazienti con valori di $\mathrm{Cr}>4.5 \mathrm{mg} / \mathrm{dl}$ sono stati mantenuti con la dieta vegetariana supplementata con aminoacidi essenziali e chetoanaloghi (alfa- kappa, Farma-Biagini, Italia). Gli schemi dietetici prevedevano un adeguato apporto calorico (30-35 $\mathrm{KCal} / \mathrm{kg} / \mathrm{die}$ ), il $57 \%$ circa di carboidrati per i pazienti in DC e il 67\% circa per quelli in DVS e il $35 \%$ e il $29 \%$ rispettivamente di lipidi.

Le caratteristiche cliniche dei pazienti 
TAB. I - CARATTERISTICHE CLINICHE DEI PAZIENTI E DEI CONTROLLI

\begin{tabular}{|c|c|c|c|c|c|}
\hline & Controlli & IRC-I & IRC-II & IRC-III & IRC-IV-DVS \\
\hline Numero (54) & 12 & 12 & 12 & 8 & 13 \\
\hline Sesso M & 8 & 5 & 8 & 4 & 9 \\
\hline Sesso F & 4 & 7 & 4 & 4 & 4 \\
\hline $\mathrm{IMC}$ media + DS & $24.2 \pm 3.11$ & $23.4 \pm 2.0$ & $23.5 \pm 2.8$ & $24.0 \pm 3.5$ & $23.1 \pm 3.5$ \\
\hline $\mathrm{Cr}$ media + $\mathrm{DS}^{\wedge}$ & $0.9 \pm 0.1$ & $1.9 \pm 0.4$ & $4.3 \pm 0.8$ & $7.8 \pm 1.7$ & $8.5 \pm 2.3$ \\
\hline Dieta DC $\# / D V S *$ & - & $8 / 0$ & $11 / 0$ & $8 / 0$ & $0 / 13$ \\
\hline
\end{tabular}

suddivisi in gruppi e dei controlli sono state schematizzate nella Tabella I.

La tolleranza glicidica è stata valutata mediante il test da carico orale di glucosio. Per la diagnosi di intolleranza glicidica sono stati seguiti i criteri indicati dal National Diabetes Data Group (NDDG) del National Institute of Health (16) secondo i quali è presente un'alterata tolleranza al glucosio quando durante le due ore del test da carico orale si ha un valore di glucosio plasmatico compreso tra 140 e $200 \mathrm{mg} / \mathrm{dl}$ e un altro $>200 \mathrm{mg} / \mathrm{dl}$.

Per il test da carico orale di glucosio i campioni di sangue sono stati prelevati a digiuno per i valori di base (glicemia, insulinemia e PTH, t 0') e a t $30,60,90$, 120,150 e 180 minuti dopo la somministrazione di glucosio $(75 \mathrm{~g})$, sia per la glicemia che per l'insulinemia.

$\mathrm{La} \mathrm{Cr}$ è stata dosata con il metodo colorimetrico e la glicemia plasmatica con il metodo enzimatico (GOD-PAP). L'insulina plasmatica e il PTH intatto sono stati dosati con i kit RIA forniti rispettivamente dalla Ditta Sorin (Italia) e dalla Ditta Nichols (USA).

I risultati delle curve glicemiche ed insulinemiche (medie \pm DS) sono stati confrontati con i corrispondenti valori del gruppo di controllo. Inoltre sono state calcolate le aree incrementali positive comprese dalle curve sia glicemiche che insulinemiche di ciascun paziente e quindi sono state confrontate con il gruppo di controllo.

Per la valutazione statistica delle differenze tra le medie è stato impiegato il test a due code di Mann-Withney per dati non appaiati. Per l'analisi della correlazione è stato applicato il test di Spear- man mentre per valutare la relazione tra due variabili è stata usata la regressione lineare semplice, considerando che le differenze erano statisticamente significative quando $\mathrm{p}<0.05$.

\section{Risultati}

La prevalenza globale di una conclamata intolleranza glicidica, secondo i criteri diagnostici del National Diabetes Data Group (16), è risultata relativamente bassa interessando solo il $14 \%$ della popolazione presa in esame $(n=45)$ e senza una significativa differenza di incidenza nell'ambito dei singoli gruppi.

Prendendo in esame i vari gruppi con diverso grado di IRC è stato osservato che il gruppo con IRC di grado lieve (IRC-I) non mostrava durante il test differenze significative rispetto ai controlli per quanto riguarda i valori glicemici (Fig. 1 ); comunque a t $120^{\prime}$ i valori di insulinemia erano aumentati $(p<0.05)$ (Fig. 2 ), mentre la produzione globale di insulina (area insulinemica) si manteneva simile al gruppo di controllo (Tab. II).

Nel gruppo con IRC di grado moderato (IRC-II) i valori di glicemia e quindi la curva glicemica erano sovrapponibili a quelli del gruppo di controllo (Fig. 1), ma a spese di un aumento significativo della secrezione insulinica $(\mathrm{p}<0.02)$ (Tab. II), con due picchi insulinemici a $t$ 90' e t $120^{\prime}$ (Fig. 2).

Peggiorando ulteriormente la funzionalità renale (gruppi IRC-III e IRC-IVDVS) si aveva uno spostamento in alto e a destra sia della curva glicemica che insulinemica con picchi glicemici ed insu- linemici a t $90^{\prime}$ e t $120^{\prime}$ per ambedue i gruppi (Figg. 1 e 2).

Quindi osservando le curve, i due gruppi avevano picchi iperglicemici e insulinemici ritardati e prolungati e a t $120^{\prime}$ ' i valori di glicemia rimanevano significativamente più alti $(\mathrm{p}<0.05)$ di quelli del gruppo di controllo (Fig. 1).

E stato possibile un confronto tra il gruppo III e IV-DVS in quanto i valori medi di $\mathrm{Cr}$ non differivano significativamente tra loro (Tab. I).

La differenza tra questi due gruppi (IRCIII e IV-DVS) stava nel fatto che nel gruppo III rispetto all'aumento significativo dell'area glicemica $(p<0.02)$ non si aveva un corrispondente aumento dell'area insulinemica; nel gruppo IV-DVS l'area glicemica era significativamente più grande $(\mathrm{p}<0.05)$ nonostante che la produzione globale di insulina fosse significativamente aumentata $(\mathrm{p}<0.05)$ (Tab. II).

I valori delle aree glicemiche e insulinemiche sono risultati correlati fra loro in modo statisticamente significativo in tutti i gruppi tranne che nel gruppo IV ( dati non riportati).

Il rapporto Area Glicemica/Area Insulinemica era simile ai controlli nel gruppo I e II, mentre risultava aumentato nei pazienti del gruppo III e IV, ma maggiormente nel gruppo mantenuto a dieta convenzionale (III). Lo stesso gruppo mostrava i più alti livelli di PTH e quindi il valore più basso del rapporto Area Insulinemica/PTH (Tab. II).

Anche dal confronto dei valori del rapporto Area Insulinemica/PTH dei due gruppi con IRC di grado avanzato si evidenziava il diverso comportamento dei 


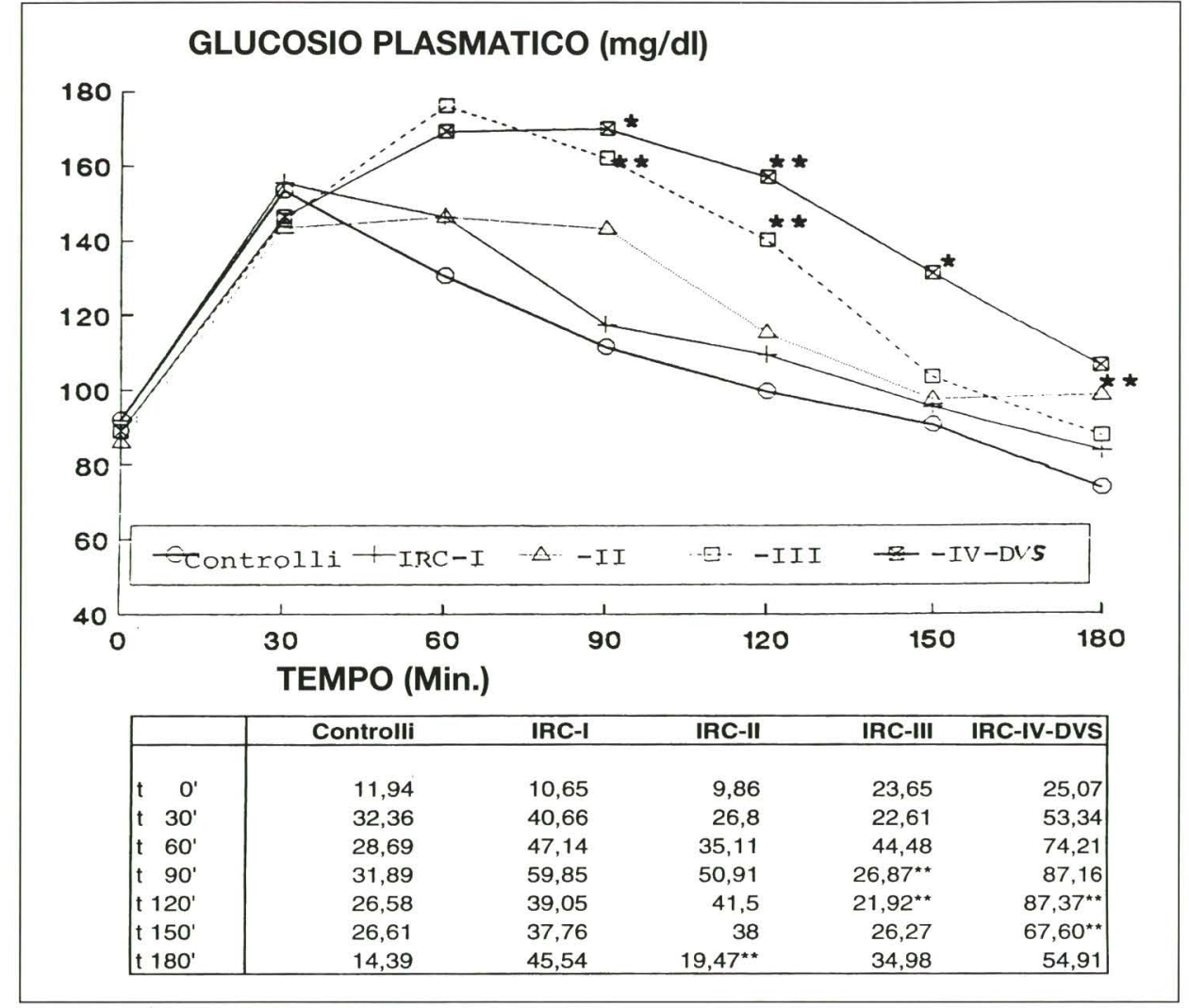

Fig. 1 - Curve glicemiche dopo carico orale di glucosio (75 g) elaborate sui valori medi dei singoli gruppi e dei controlli. In basso sono riportati $i$ valori delle relative deviazioni standard.

$* p<0.05 ; * * p<0.02$. mici in genere sono comunque normali o lievemente aumentati e al test da carico orale di glucosio solo alcuni mostrano una chiara intolleranza glicidica. Infatti nel presente studio la diagnosi di intolleranza glicidica secondo i criteri del NDDG (16), è stata fatta solo nel $14 \%$ dell'intera popolazione esaminata, quindi relativamente rara.

Effettivamente per quanto riguarda il metabolismo del glucosio nella IRC si possono distinguere due gruppi di pazienti: il primo con aumentata secrezione insulinica tale da conservare la tolleranza glicidica; il secondo con livelli di insulina normali o bassi in cui si manifesta l'intolleranza glicidica $(1,6)$.

Un aumento della secrezione insulinica media era comune a tutti i gruppi di pazienti studiati, con IRC di vario grado, ma una alterata tolleranza glicidica era più evidente in quelli con IRC grave, indipendentemente dal regime dietetico seguito, dieta ipoproteica convenzionale oppure vegetariana supplementata.

Questi risultati suggeriscono che un'alterata sensibilità all'insulina è presente fin dagli stadi precoci della malattia.

Inoltre i valori di insulina aumentavano tendenzialmente con il declino della funzionalità renale in accordo con l'ipotesi pazienti mantenuti a dieta supplementata rispetto ai pazienti mantenuti a dieta convenzionale: infatti il gruppo IV-DVS aveva un rapporto Area Insulinemica/ PTH molto più alto rispetto al gruppo III $(p<0.01)$ e molto simile al gruppo di controllo, con valori di PTH molto più bassi $(\mathrm{p}<0.001)$ (Tab. II).

La Figura 3 mostra la relazione inversa riscontrata tra il rapporto Area Insulinemica/PTH e la $\mathrm{Cr}$ in tutti i gruppi. Nella figura sono riportati separatamente $i$ dati relativi al IV gruppo per evidenziare il diverso comportamento rispetto al gruppo III.

\section{Discussione}

Nella IRC è stata ampiamente dimostrata una intolleranza glicidica, come conseguenza di una resistenza tissutale periferica, principalmente del tessuto muscolare scheletrico, all'azione ipoglicemizzante dell'insulina e di una inadeguata secrezione dell'ormone (1-3,7).

L'esperienza clinica dimostra che i livelli di glicemia a digiuno dei pazienti ure-

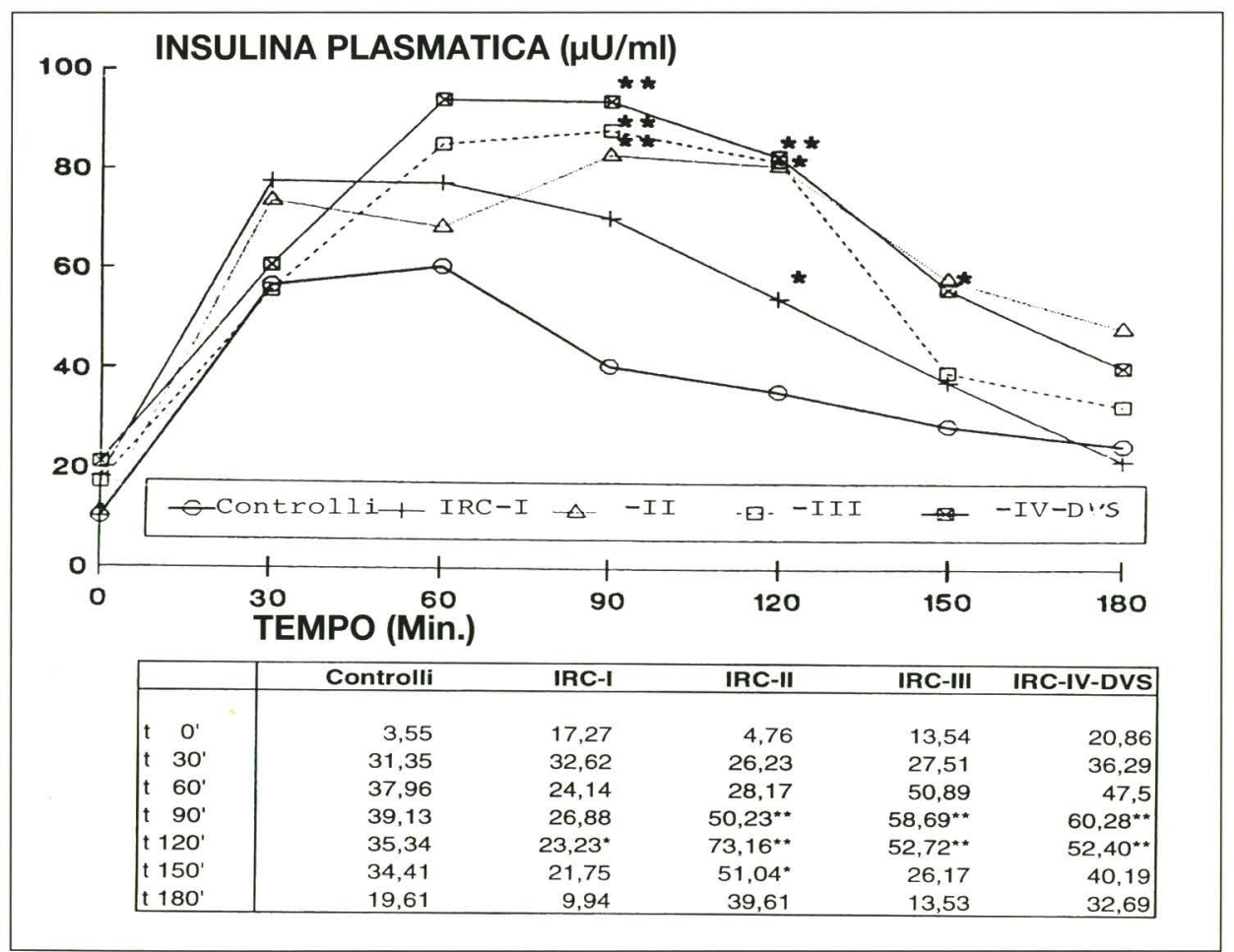

Fig. 2 - Curve insulinemiche dopo carico orale di glucosio (75g) elaborate sui valori medi dei singoli gruppi e dei controlli. In basso sono riportati $i$ valori delle relative deviazioni standard. $* p<0.05 ; * * p<0.02$. 
TAB. II - VALORI DELL'AREA GLICEMICA (AG), DELL'AREA INSULINEMICA (AI), DEL RAPPORTO AG/AI E AI/PTH E DEL PTH DEI PAZIENTI E DEI CONTROLLI (MEDIE + DS)

\begin{tabular}{lccccc}
\hline & Controlli & IRC-I & IRC-II & IRC-III & IRC-IV-DVS \\
\hline AG & $71 \pm 48$ & $100 \pm 71$ & $121 \pm 68$ & $150 \pm 37 * *$ & $173 \pm 128^{*}$ \\
AI & $89 \pm 68$ & $117 \pm 49$ & $9 \pm 89^{* *}$ & $132 \pm 97$ & $161 \pm 102^{*}$ \\
AG/AI & $0.91 \pm 0.70$ & $0.9 \pm 0.66$ & $0.89 \pm 0.54$ & $1.76 \pm 1.18$ & $1.28 \pm 0.88$ \\
AI/PTH & $3.41 \pm 3.15$ & $2.35 \pm 1.68$ & $1.82 \pm 2.55^{* *}$ & $0.80 \pm 0.77 * * *$ & $3.55 \pm 3.83^{\mathrm{a}}$ \\
PTH (ng/ml) & $34.53 \pm 19$ & $69.7 \pm 50.1$ & $163.3 \pm 109^{* * * *}$ & $322.2 \pm 384.2^{* * * *}$ & $73 \pm 66.6^{\mathrm{b}}$ \\
\hline
\end{tabular}

Significatività statistica vs controlli:

Significatività statistica IRC-III vs IRC-IV-DVS:

$$
\begin{aligned}
& * \mathrm{p}<0.05 \\
& * * \mathrm{p}<0.02 \\
& * * * \mathrm{p}<0.01 \\
& * * * * \mathrm{p}<0.002
\end{aligned}
$$

$$
\begin{aligned}
& { }^{\mathrm{a}} \mathrm{p}<0.01 \\
& \mathrm{p}<0.001
\end{aligned}
$$

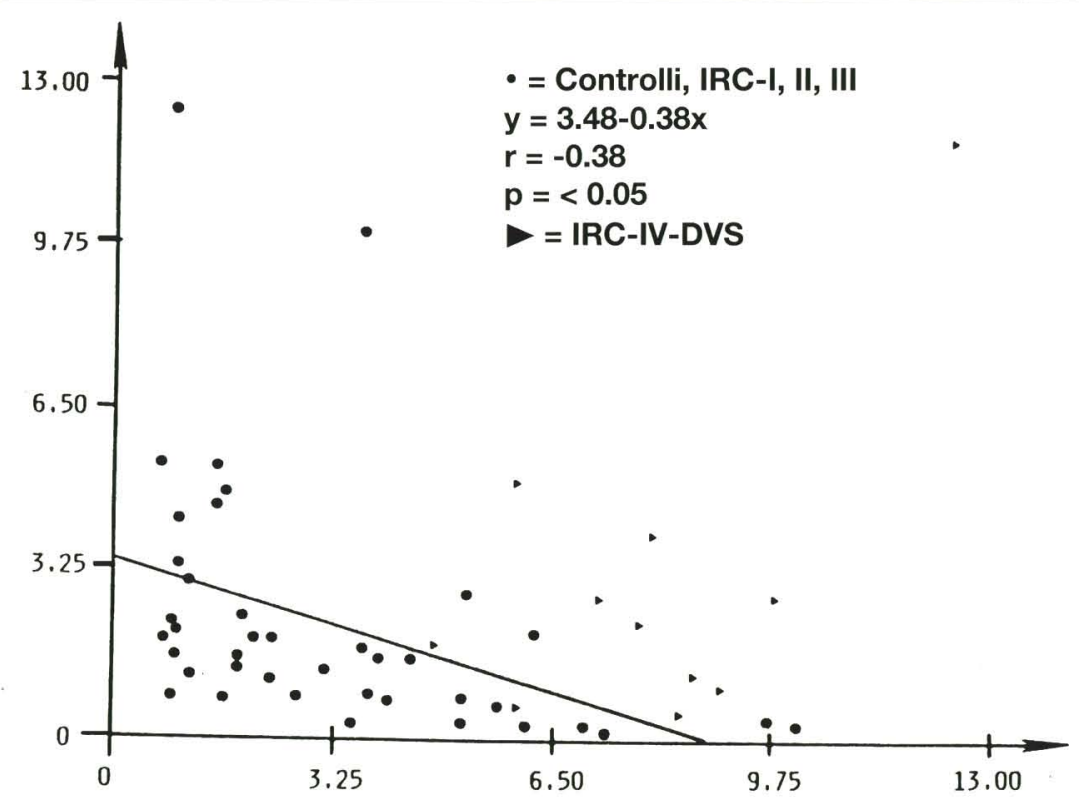

Fig. 3 - La figura mostra la relazione tra i valori del rapporto Area InsulinemicalPTH e quelli della Cr.

che con il peggiorare della IRC si accumulerebbero una $o$ più tossine uremiche responsabili della resistenza tissutale periferica all'insulina (18). A favore di questa ipotesi c'è la dimostrazione che l'intolleranza glicidica è parzialmente corretta dalla terapia dialitica, quindi si tratterebbe di fattori sierici dializzabili che potrebbero compromettere l'azione biologica dell'insulina (19-23).

Nonostante l'aumento dei livelli medi di insulina osservato in tutti i gruppi, i pazienti con IRC di grado avanzato a dieta convenzionale (IRC-III) presentavano livelli più bassi rispetto al gruppo con IRC di grado moderato (IRC-II) e del gruppo mantenuto a dieta vegetariana supplementata con chetoanaloghi (IRC-IVDVS). Questo diverso comportamento potrebbe essere in parte spiegato con gli alti livelli di PTH riscontrati in questi pazienti (gruppo IRC-III).

Infatti l'iperparatiroidismo secondario allo stato uremico sarebbe responsabile di un'alterata risposta delle beta-cellule pancreatiche (5-9). Studi sperimentali hanno dimostrato che la paratiroidectomia nel cane uremico è in grado di prevenire l'intolleranza glicidica (10) e la correzione dell'iperparatiroidismo in bambini affetti da IRC ha portato ad un aumento della secrezione insulinica e quindi alla cura dell'intolleranza glicidica $(8,12)$.

D'altra parte però nel gruppo mantenuto con dieta vegetariana supplementata (gruppo IRC IV-DVS) si sono riscontrati valori significativamente più bassi di PTH rispetto al corrispondente gruppo a dieta convenzionale (IRC-III) valori di insulina tendenzialmente più alti, con un rapporto Area Insulinemica/PTH simile al gruppo dei controlli; tuttavia questo non era sufficiente a mantenere in tutti una normale risposta al test da carico orale di glucosio.

Tali risultati non confermerebbero l'influenza positiva della dieta vegetariana supplementata sulla tolleranza glicidica nell'uremia come affermato in altri studi $(13,17,19)$. Tale influenza è stata attribuita in parte ad un miglioramento della sensibilità all'insulina dovuto ad una ridotta produzione e/o accumulo di tossine uremiche $(17,19)$ ed in parte al miglior controllo dell'iperparatiroidismo secondario con conseguente aumento di secrezione insulinica $(8,12)$. È stato dimostrato che questi meccanismi possono intervenire ed influenzare la tolleranza glicidica contemporaneamente e indipendentemente $(8,19)$.

Sebbene non si possa valutare l'esatta patogenesi dell'alterato metabolismo del glucosio nei pazienti uremici solo sulla base del test da carico orale, l'alterata risposta al test in presenza di inadeguati livelli di insulina e di un iperparatiroidismo lieve nel gruppo mantenuto a dieta vegetariana supplementata non suggerisce comunque né un miglioramento del metabolismo del glucosio e quindi della sensibilità all'insulina né un ruolo deter- 
minante del PTH dell'alterazione del metabolismo glicidico in questo gruppo di pazienti.

E probabile che altri fattori, diversi dal PTH, possano condizionare il rilascio di insulina dalle beta-cellule pancreatiche, e.g. i livelli di calcio totale o ionizzato, $\mathrm{i}$ fosfati e la 1,25(OH)2D3 $(6,8,12)$. Alcuni studi infatti hanno dimostrato recentemente che la somministrazione di boli di 1,25 $(\mathrm{OH}) 2 \mathrm{D} 3$ a pazienti uremici in terapia dialitica periodica, sono in grado di correggere l'intolleranza glicidica inducendo un aumento di secrezione insulinica, indipendentemente dai valori di PTH $(21,23)$.

In conclusione i risultati di questo studio suggeriscono che:

1) un'alterata tolleranza glicidica, dovuta ad uno stato di resistenza tissutale periferica all'insulina, è presente fino dalle fasi più precoci della IRC;

2) una chiara intolleranza glicidica, secondo i comuni criteri diagnostici, è probabilmente rara nell'uremia, in quanto l'aumento della secrezione insulinica supera la resistenza tissutale periferica. Con il peggiorare della malattia la secrezione insulinica diventa inadeguata e comunque la risposta al test da carico orale di glucosio risulta alterata;

3) i pazienti uremici mantenuti a dieta vegetariana supplementata con aminoacidi essenziali e chetoanaloghi non mostrano un comportamento al test migliore rispetto a quelli mantenuti a dieta ipoproteica convenzionale;

4) probabilmente altri fattori, oltre il PTH, potrebbero spiegare l'alterazione del rilascio di insulina dalle cellule pancreatiche nell'uremia cronica.

\section{Riassunto}

È stata studiata la risposta al test da carico orale di glucosio in 45 pazienti (26 M e $19 \mathrm{~F}$, età media $60 \pm 10$ anni) affetti da insufficienza renale cronica (IRC) di vario grado, divisi in quattro gruppi a seconda dei valori di creatinina (Cr), (IRC -I: Cr 1.5 - $\leq 3 \mathrm{mg} / \mathrm{dl}$; IRC-II: $\mathrm{Cr}>3$ - $\leq$ $6 \mathrm{mg} / \mathrm{dl}$; IRC-III: $\mathrm{Cr}>6 \mathrm{mg} / \mathrm{dl}$ ). I pazienti di questi primi tre gruppi sono stati mantenuti a dieta ipoproteica convenzionale, mentre un quarto gruppo (IRC IV-DS: $\mathrm{Cr}>4.5 \mathrm{mg} / \mathrm{dl}$ ) a dieta vegetariana supplementata con aminoacidi essenziali e chetoanaloghi. È stata valutata l'influenza del progressivo deteriora- mento della funzione renale, dell'iperparatiroidismo secondario e del diverso regime dietetico (gruppo IRC-III -valori medi di Cr $7.82 \pm 1.73 \mathrm{mg} / \mathrm{dl}$ - vs IRCIV-DS - valori medi di $\mathrm{Cr} 8.47 \pm 2.27$ $\mathrm{mg} / \mathrm{dl}-$ ) sulla tolleranza glicidica e sulla secrezione insulinica, confrontando i risultati ottenuti con un gruppo di controllo costituito da 12 soggetti sani ( $8 \mathrm{M}$ e 4 $\mathrm{F}$, età media $47 \pm 8$ anni). È stato riscontrato un aumento della secrezione insulinica fin dagli stadi precoci della IRC, secondaria alla resistenza tissutale periferica all'azione dell'ormone, che riusciva a mantenere la tolleranza glicidica. Con il peggiorare della funzione renale si assisteva ad uno spostamento in alto e a destra sia della curva glicemica che insulinemica, quindi la secrezione insulinica diventava inappropriata e non riusciva più a superare lo stato di resistenza periferica. I pazienti mantenuti a dieta vegetariana supplementata non hanno mostrato un comportamento migliore rispetto a quelli in dieta ipoproteica convenzionale, nonostante i valori più bassi di paratormone (PTH) e gli alti valori di insulinemia. II PTH non sembra essere un fattore critico nell'alterata tolleranza glicidica nei pazienti uremici.

\section{BIBLIOGRAFIA}

1.

DeFronzo RA, Andres R, Edgar P, et al. Carbohydrate metabolism in uremia: a review. Medicine 1973; 52: 469-81.

2. DeFronzo RA, Tobin JD, Rowe JW, et al. Glucose intolerance in uremia. Quantification of pancreatic beta-cell sensitivity to glucose and tissue sensitivity to insulin. J Clin Invest 1978; 62: 425-35.

3. Graf H, Prager R, Kovarik J, et al. Glucose metabolism and insulina sensitivity in patients on chronic hemodialysis. Metabolism 1985; 34: 974-7.

4. DeFronzo RA, Tobin J, Andres $R$, et al. The glucose clamp technique. A method for the quantification of beta-cell sensitivity to glucose an of tissue sensitivity to insulin. Am J Physiol 1979; 237: E21423.

5. Mak RHK. Insulin secretion in uremia: effect of parathyroid hormon and vitamin D metabolites. Kidney Int 1979; 36 (suppl 127): S277-30.

6. Mak RHK, DeFronzo RA. Glucose and insulin metabolism in uremia. Nephron 1992; 61: 377-82.

7. DeFronzo RA, Alvestrand A. Glucose intolerance in uremia: site and mechanism. Am J Clin Nutr 1980; 33: 1438-45.

8. Mak RHK, Turner C, Haycook $\mathrm{G}$ et al. Secondary hyperparathyroidism and glucose intolerance in children with uremia. Kidney Int 1983; 24 (suppl 16): S128-33.

9. Massry SG. Uremia, parathyroid hormone and carbohydrate intolerance. Contr Nephrol 1986; 150: 96-108.

10. Akmal M, Massry SG, Goldstein DA, et al. Role of parathyroid hormone on the glucose intolerance in chronic renal failure. J Clin Invest 1985; 75: 1037-44. 
11.

Fadda GZ, Akmal M, Premdas $\mathrm{FH}$, et al. Insulin release from pancreatic islets: effects of CRF and excess PTH. Kidney Int 1988; 33: 166-72.

12. Mak RHK, Bettinelli A, Turner $\mathrm{C}$, et al. The influence of hyperparathyroidism on glucose metabolism in uremia. J Clin Endocrinol Metab 1985; 60: 229-33.

13. Giovannetti S. Supplemented diet for severe chronic renal failure: some controversial points. Contr Nephrol 1988; 75: 147-54.

14. Barsotti G, Navalesi R, Giampietro O, et al. Effects of vegetarian supplemented diet on renal function, proteinuria and glucose metabolism in patients with overt diabetic nephropathy and renal insufficiency. Contr Nephrol 1988; 65: 87-94.

15. Grutzmahcer P, Radkte HW, Schifferecker E, et al. Early changes of plasma lipid status and glucose tolerance during course of chronic renal failure. Contr Nephrol 1984; 41: 332-6.

16. National Diabetes Data Group. Classification and diagnosis of diabetes mellitus and other categories of glucose intolerance. Diabetes 1979; 28: 1039-59.

17. Aparicio M, Gin H, Potaux L, et al. Effect of ketoacid diet on glucose tolerance and tissue insulin sensitivity. Kidney Int 1989; 36 (suppl 27): S231-5.

18. McLabel ML, Izzo MS, Lockwood DH. Characterization and partial purification of a factor from uremic human serum that induces insulin resistance. J Clin Invest $1985 ; 75: 391-6$.

19. Mak RHK, Turner C, Thomson T, et al. The effect of low protein diet with aminoacid/ketoacid supplements on glucose metabolism in children with uremia. J Clin Endocrinol Metab 1986; 63: 985-9.

20. Synder D, Pulido LB, Kagan A. Dietary reversal of the carbohydrate intolerance in uremia. Proc EDTA 1968; 5: 205-9.
21. Mak RHK. Intravenous 1,25 dihydroxycholecalciferol corrects glucose intolerance in hemodialysis patients. Kidney Int 1992; 41: 104954.

22. Turk S, Yeksan M, Tamer N, et al. Effect of 1,25(OH)2D3 treatment on glucose tolerance in uremia. Nephrol Dial Transplant 1992; 7: 1207-12.

23. Quesada JM, Martin-Malo A, Santiago J, et al. Effects of calcitriol on insulin secretion in uremia. Nephrol Dial Transplant 1990; 5: 1013-7. 